\title{
Fatores desencadeantes do estresse psicológico em residentes não médicos: revisão
} integrativa

\section{Triggering factors of psychological stress in non-medical residents: an integrative review}

\section{Factores que desencadenan el estrés psicológico en residentes no médicos: revisión integradora}

\author{
Recebido: $30 / 08 / 2020$ \\ Aprovado: 05/03/2021 \\ Publicado: 14/10/2021
}

\author{
Gabriela França Rosinha ${ }^{1}$ \\ Suzel Regina Ribeiro Chavaglia ${ }^{2}$ \\ Fabiana Cristina Pires ${ }^{3}$ \\ Rosali Isabel Barduchi Ohl ${ }^{4}$ \\ Lúcia Aparecida Ferreira ${ }^{5}$ \\ Álvaro da Silva Santos 6
}

Esta é uma revisão integrativa realizada em 2020, considerando o período de 2005 a 2019, com o objetivo de identificar evidências que descrevam os fatores desencadeantes do estresse psicológico em residentes não médicos nos serviços de saúde. A questão norteadora foi: Quais as evidências científicas que descrevem o estresse psicológico em residentes não médicos nos serviços de saúde? Considerou-se as bases LILACS, CINAHL, PubMed/Medline, Scopus e Web of Science, produções em português, inglês e espanhol, com os principais descritores: estresse psicológico, internato não médico e combinações. Inicialmente identificou-se 2540 artigos, mas destes, apenas cinco foram incluídos, três nacionais e dois internacionais. Dentre os fatores mais citados que desencadeiam o estresse destacaram-se: a sobrecarga de trabalho, o atendimento a pacientes graves, exaustão emocional e privação de sono. Assim, poucas são as evidências científicas sobre os fatores desencadeantes do estresse psicológico nos residentes não médicos, portanto, é indispensável novos estudos científicos bem delineados, com bom nível de evidência, que explorem a temática com mais profundidade.

Descritores: Estresse psicológico; Internato não médico; Pessoal de saúde; Serviços de saúde.

This is an integrative review carried out in 2020, considering the period from 2005 to 2019. It aimed to identify evidence that describes triggering factors of psychological stress in non-medical residents in health services. The guiding question was: What is the scientific evidence that describes psychological stress in non-medical residents in health services? The databases LILACS, CINAHL, PubMed/Medline, Scopus and Web of Science, productions in Portuguese, English and Spanish were considered, with the main descriptors: estresse psicológico (psychological stress), internato não médico (non-medical internship) and combinations. Initially, 2540 articles were identified, but of these only five were included, three from Brazil and two from other countries. Among the most mentioned factors that trigger stress were: work overload, care for critically ill patients, emotional exhaustion and sleep deprivation. Thus, there is little scientific evidence on the triggering factors of psychological stress in non-medical residents, therefore, further well-designed scientific studies, with a good level of evidence, that explore the subject in greater depth, are essential.

Descriptors: Stress, Psychological; Internship, Nonmedical; Health personnel; Health services.

Esta es una revisión integradora realizada en 2020, considerando el periodo comprendido entre 2005 y 2019, con el objetivo de identificar las evidencias que describen los factores desencadenantes del estrés psicológico en los residentes no médicos de los servicios de salud. La pregunta guía fue: ¿Cuáles son las evidencias científicas que describen el estrés psicológico en los residentes no médicos de los servicios de salud? Se consideraron las bases LILACS, CINAHL, PubMed/Medline, Scopus y Web of Science, trabajos en portugués, inglés y español, con los principales descriptores: estrés psicológico, pasantías no médicas y combinaciones. Inicialmente se identificaron 2.540 artículos, pero de ellos sólo se incluyeron cinco, tres nacionales y dos internacionales. Entre los factores más mencionados que desencadenan el estrés destacan: la sobrecarga de trabajo, el cuidado de pacientes en estado crítico, el agotamiento emocional y la falta de sueño. Por lo tanto, hay poca evidencia científica sobre los factores desencadenantes del estrés psicológico en los residentes no médicos, entonces, son esenciales nuevos estudios científicos bien diseñados y con un buen nivel de evidencia que exploren el tema en mayor profundidad.

Descriptores: Estrés psicológico; Internado no médico; Personal de salud; Servicios de salud.

1. Enfermeira. Especialista em Urgência e Trauma. Mestranda do Programa de Pós Graduação em Atenção à Saúde da Universidade Federal do Triângulo Mineiro (PPGAS/UFTM), Uberaba, MG, Brasil. ORCID: 0000-0002-1061-5805 E-mail: gabrielarosinha@hotmail.com

2. Enfermeira. Especialista em Gestão em Saúde. Mestre, Doutora e Pós Doutora em Enfermagem. Professora Titular do PPGAS e do Curso de Graduação em Enfermagem (CGE) da UFTM, Uberaba, MG, Brasil. ORCID: 0000-0001-7033-0185 E-mail: suzel.ribeiro@yahoo.com.br

3. Enfermeira. Especialista em Urgência e Emergência. Mestranda do PPGAS/UFTM, Uberaba, MG, Brasil. ORCID: 0000-0002-8524-1449 E-mail: enfermagem.pires@gmail.com

4. Enfermeira. Especialista em Enfermagem Médico Cirúrgica. Especialista em Enfermagem em Saúde Mental e Psiquiátrica. Mestre e Doutora em Enfermagem. Professora Associada do Curso de Enfermagem da Universidade Federal de São Paulo, SP, Brasil. ORCID: 0000-0002-0760-2173 E-mail: rosaliohl@hotmail.com

5. Enfermeira. Especialista em Educação Profissional em Enfermagem. Mestre em Enfermagem Psiquiátrica. Doutora em Enfermagem. Professora Associada do PPGAS e coordenadora do CGE da UFTM, Uberaba, MG, Brasil. ORCID: 0000-0001-6469-5444 E-mail: lap2ferreira@yahoo.com.br

6. Enfermeiro. Psicanalista. Especialista em Saúde Pública. Especialista em Enfermagem Médico Cirúrgica. Mestre me Administração em Serviços de Saúde. Doutor em Ciências Sociais. Pós Doutor em Serviço Social. Professor Associado do PPGAS e do CGE da UFTM, Uberaba, MG, Brasil. ORCID: 0000-0002-8698-5650 E-mail: alvaroenf@hotmail.com 


\section{INTRODUÇÃO}

A

Residência Multiprofissional em Saúde (RMS) é instituída mediante a Lei no 11.129/2005 como uma formação em nível de Pós-graduação latu sensu com duração estabelecida de dois anos e carga horária semanal de 60 horas, que possui como principal característica o trabalho em campo, destacando-se como modalidade de formação profissional para o Sistema Único de Saúde (SUS) ${ }^{1-4}$.

Os programas de residência médica, bem como as residências multiprofissionais, são caracterizados por um processo de formação intensiva e de assistência em níveis progressivos, que resulta em aspectos que podem atuar como fatores estressores, dentre eles as relações interpessoais, a carga horária exaustiva, a responsabilidade sobre o cuidado integral ao paciente e estabelecer os limites da sua identidade pessoal e profissional de ensino/aprendizagem e ao ambiente profissional5-8.

Os profissionais da saúde, ao longo da carreira e principalmente durante o período de residência, enfrentam ainda situações como: privação do sono, sobrecarga de trabalho, problemas relacionados ao processo de ensino e aprendizagem e ao ambiente profissional, estando sujeitos ao surgimento do estresse físico e psicológico ${ }^{8,9}$.

0 estresse é definido como um conjunto de reações e estímulos do organismo com componentes físicos, psíquicos e hormonais que o levam a distúrbios de desiquilíbrio diante de uma situação desafiadora ${ }^{10}$. No ambiente de trabalho, o estresse pode ocorrer pela demanda excessiva e escassez de recursos de enfrentamento, representando um risco para saúde física e mental desses profissionais, bem como para os pacientes que são por eles atendidos 8,11.

São descritos como sinais físicos do estresse o aumento da sudorese, tensão muscular, taquicardia, hipertensão, aperto da mandíbula, ranger de dentes, hiperatividade, náuseas, mãos e pés frios; e em termos psicológicos é evidenciado ansiedade, tensão, angústia, insônia, alienação, dificuldades interpessoais, dúvidas quanto a si próprio, preocupação excessiva, inabilidade de concentrar-se em outros assuntos que não o relacionado ao sofrimento, dificuldade de relaxar, ira e hipersensibilidade emotiva ${ }^{12}$.

Tais sinais físicos e psicológicos do estresse psicológico interferem no processo de viver e adoecer humano gerando desgastes, insatisfações e sofrimentos psíquicos que por sua vez, afetam condutas de saúde individuais e coletivas, qualidade de vida, sensação de bem-estar e produtividade, alterando as relações do indivíduo na área familiar, no campo dos estudos, do trabalho e das interações sociais ${ }^{13,14}$.

Residentes de diferentes áreas profissionais da saúde ocupam simultaneamente a posição de estudantes e de profissionais, enfrentando características próprias desse cenário como a sobrecarga curricular e de trabalho, a ausência de tempo à vida acadêmica e pessoal, e as incertezas quanto à futura inserção profissional, falta de reconhecimento como membros da equipe de saúde e as dificuldades de relacionamento entre os membros do grupo, fatores estes que são capazes de afetar o seu ajustamento psicossocial ${ }^{4,15}$.

Nesse contexto de estudo e trabalho relacionado aos programas de RMS, o estresse e a ansiedade são associados a indicadores negativos das condutas de saúde como alterações comportamentais, falta de atenção e rendimento, aumento do uso de drogas e álcool, alterações de peso, não desenvolvimento de atividade física, hábitos de dieta inadequados e estilos de vida não saudáveis em geral, indicando válvulas de escape sociais ${ }^{13,16,17}$.

É indispensável a investigação dos fatores desencadeadores do estresse psicológico com vistas a implantar estratégias para prevenir e minimizar o estresse psicológico no ambiente da RMS, beneficiando a saúde física e mental dos residentes não médicos e melhorando a qualidade de vida no trabalho ${ }^{14,18,19}$.

Em vista da necessidade de reconhecer os fatores desencadeantes do estresse psicológico e a implementação de estratégias de prevenção, são esperados dos programas de residência em saúde ações de prevenção e minimização do estresse e desenvolvimento de um ambiente de trabalho menos desgastante, com impacto na produtividade e qualidade do trabalho ${ }^{14,18,19}$. 
Assim, o presente estudo teve como objetivo identificar evidências que descrevam os fatores desencadeantes do estresse psicológico em residentes não médicos nos serviços de saúde.

\section{MÉTODO}

Esta é uma revisão integrativa ${ }^{20}$ que possibilita a síntese e análise do conhecimento científico já produzido sobre determinada temática; no presente caso, acerca do estresse psicológico em residentes não médicos.

Utilizou-se as recomendações da ferramenta Preferred Reporting Items for Systematic Reviews and Meta-Analysis (PRISMA) ${ }^{21}$. Para tanto, foram realizadas seis etapas, sendo elas: a) definição da questão de pesquisa; b) estabelecimento dos critérios de inclusão e exclusão; c) definição das informações que serão extraídas dos estudos; d) avaliação dos estudos incluídos; e) interpretação dos resultados e f) síntese dos dados ${ }^{20}$.

A primeira etapa foi composta pela identificação do tema sobre o estresse psicológico em residentes não médicos, e pela seleção da pergunta de pesquisa usando a estratégia Population - Interest Phenomenon - Context (PICo), sendo $\mathrm{P}=$ população, $\mathrm{I}=$ fenômeno de interesse e Co = contexto do estudo 22 .

Tendo a estratégia "PICo" como referência, neste estudo o acrônimo "P" foi representado pelos residentes não médicos; o "I" representado pela identificação de estudos acerca do estresse psicológico; e "Co" representado pelos serviços de saúde, construindo-se a seguinte pergunta norteadora: Quais as evidências científicas que descrevem o estresse psicológico em residentes não médicos nos serviços de saúde?

Na segunda etapa foram definidos os critérios de inclusão e exclusão. Sendo incluídos estudos primários que respondessem à pergunta de pesquisa, disponíveis na íntegra, publicados em português, inglês ou espanhol, no período de 2005 a 2020, uma vez que o Programa de Residências Multiprofissionais não médicas foi iniciado em 2005.

Excluíram-se estudos do tipo revisão, teses, dissertações, artigos de opinião, comentários, ensaios, notas prévias, manuais, livros, capítulos de livros, obituários e artigos que abordassem o tema estresse em residentes médicos de forma isolada ou em outro público que não estivesse inserido em um programa de residência na área da saúde.

Foram utilizadas as bases de dados: Literatura Latino Americana e do Caribe em Ciências da Saúde (LILACS), Cumulative Index to Nursing and Allied Health Literature (CINAHL), US National Library of Medicine National Institutes Database Search of Health (PubMed), Medical Literature Analysis and Retrieval System Online (Medline), Scopus e Web of Science (WOS).

A coleta ocorreu no mês de fevereiro de 2020, por meio dos descritores em saúde disponíveis no Portal Regional da Biblioteca Virtual da Saúde (BVS) - Descritores em Ciências da Saúde (DeCS) e Medical Subject Headings (MeSH). Por meio de estratégias de buscas específicas, validadas por uma bibliotecária. Para cada base de dados, os descritores foram combinados com operadores booleanos "AND" e "OR" como apresentado no Quadro 1.

Ainda nesta segunda etapa, procedeu-se primeiramente à leitura de títulos e resumos, viabilizada pelo aplicativo de revisão Rayyan, virtual e gratuito, que agiliza o processo inicial de seleção dos artigos, de forma segura e confiável ${ }^{23}$, por dois revisores separadamente, havendo 23 divergências. As divergências foram encaminhadas para um terceiro revisor, responsável por decidir a inclusão ou exclusão, baseando-se nos critérios previamente definidos. 
Quadro 1: Estratégia de busca para cada base de dados e descritores utilizados. Uberaba, MG, 2020.

\begin{tabular}{|c|c|c|}
\hline $\begin{array}{l}\text { BASES DE } \\
\text { DADOS }\end{array}$ & DESCRITORES & ESTRATÉGIA DE BUSCA \\
\hline LILACS & $\begin{array}{l}\text { "Internato não Médico"; } \\
\text { Nonmedical"; "Internship, } \\
\text { "Estresse } \\
\text { Psicológico"; }\end{array}$ & $\begin{array}{l}\text { "“Estresse Psicológico" OR "Stress, Psychological" } \\
\text { OR "Estrés Psicológico" AND "Internato não } \\
\text { Médico" OR "Internship, Nonmedical" OR } \\
\text { "Internado no Médico") }\end{array}$ \\
\hline CINAHL & $\begin{array}{l}\text { "Internship, } \quad \text { Nonmedical"; } \\
\text { Personnel"; "Stress, Psychological". }\end{array}$ & $\begin{array}{l}\text { ("Stress, Psychological" AND "Internship, } \\
\text { Nonmedical" OR "Health Personnel") }\end{array}$ \\
\hline $\begin{array}{l}\text { PubMed }{ }^{\circledR} / \\
\text { Medline }\end{array}$ & $\begin{array}{l}\text { "Internship, } \quad \text { Nonmedical"; } \\
\text { Personnel"; "Stress, Psychological". }\end{array}$ & $\begin{array}{l}\text { ("Stress, Psychological" } \text { AND "Internship, } \\
\text { Nonmedical" OR Health Personnel") }\end{array}$ \\
\hline $\begin{array}{l}\text { Web Of } \\
\text { Science }\end{array}$ & $\begin{array}{lll}\text { "Internship, } & \text { Nonmedical"; } & \text { "Health } \\
\text { Personnel"; "Stress, Psychological”. } & \\
\end{array}$ & $\begin{array}{l}\text { "Stress, Psychological" AND "Internship, } \\
\text { Nonmedical" OR “Health Personnel") }\end{array}$ \\
\hline Scopus & 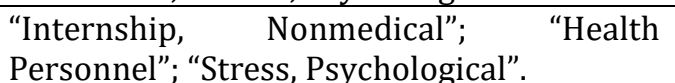 & $\begin{array}{l}\text { ("Stress, Psychological" AND "Internship, } \\
\text { Nonmedical" AND "Health Personnel") }\end{array}$ \\
\hline
\end{tabular}

A terceira etapa definiu as informações a serem extraídas de cada estudo, e, para tal, utilizou-se alguns dos critérios do instrumento de Ursi e Galvão ${ }^{24}$ com o propósito de extrair, organizar e sumarizar as informações e facilitar a formação do banco de dados. São eles: autores, ano de publicação, título da pesquisa, periódico, objetivo, método, resultados/ conclusões e nível de evidência.

Para classificar o nível de evidência dos estudos selecionados, foram utilizadas as categorias da Agency for Healthcare Research and Quality (AHRQ), que abrangem seis níveis: nível 1: meta-análise de múltiplos ensaios clínicos controlados e randomizados; nível 2: estudos individuais com delineamento experimental; nível 3: estudos quase experimentais; nível 4: estudos descritivos (não experimentais) ou abordagem qualitativa; nível 5: relatos de caso ou experiência; nível 6: opiniões de especialistas 25.

Na quarta etapa ocorreu a leitura individual na íntegra dos estudos incluídos, a avaliação crítica dos artigos em relação ao objeto de estudo desta pesquisa, para posterior interpretação dos resultados pelos revisores e síntese de conhecimento.

\section{RESULTADOS}

A Figura 1 apresenta a sequência de seleção das produções. Foram selecionados cinco (5) estudos, publicados no período entre 2005 e 2020 detalhados no Quadro 2.

A partir da leitura e síntese dos estudos selecionados, foi possível evidenciar os fatores desencadeantes do estresse psicológico em residentes não médicos. 
Figura 1: Seleção de estudos segundo o PRISMA21, Uberaba, Brasil, 2020.

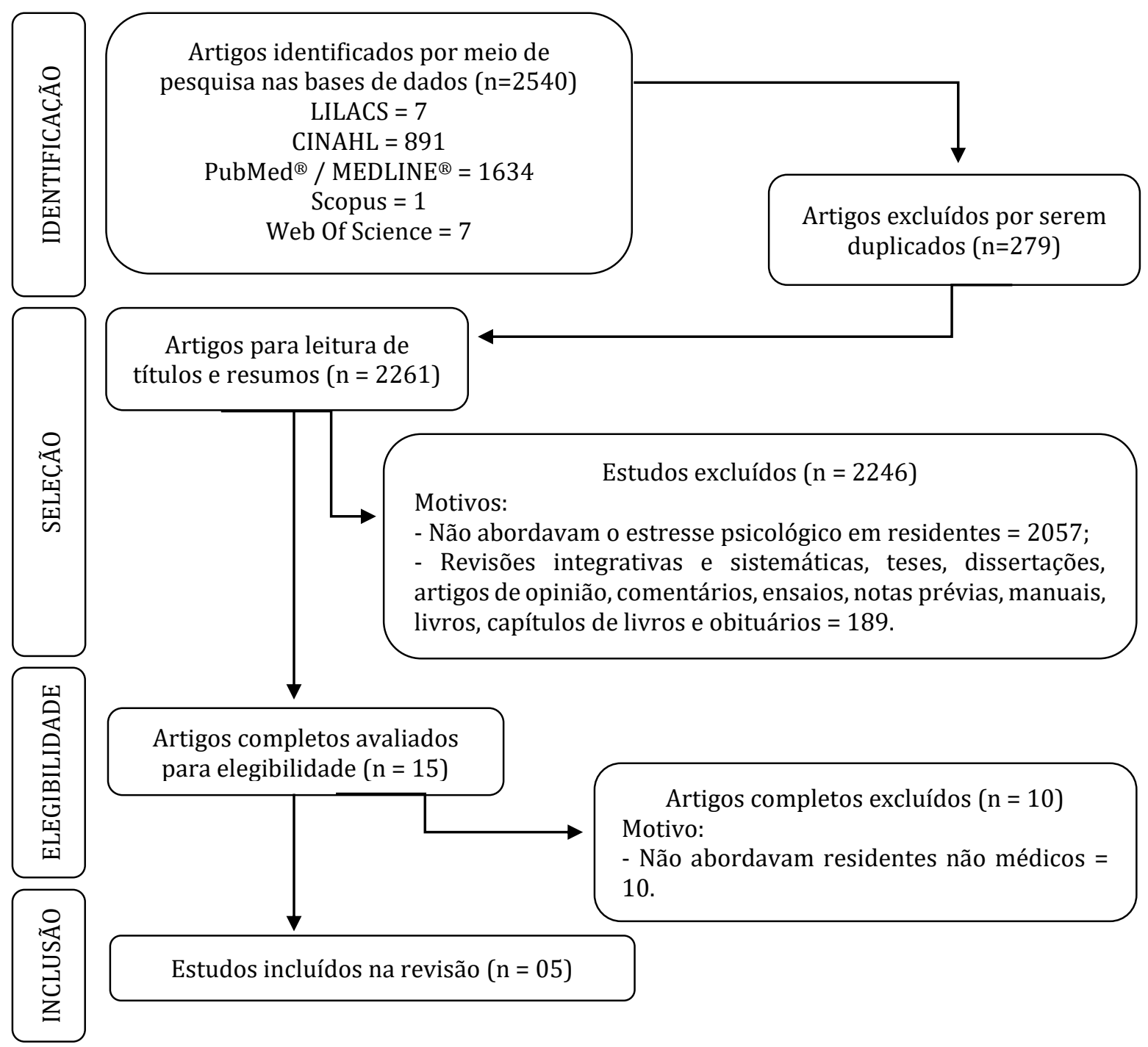

*CINAHL: Cumulative Index to Nursing and Allied Health Literature; LILACS: Literatura Latino-Americana e do Caribe em Ciências da Saúde. 
Quadro 2: Caracterização dos estudos incluídos na revisão integrativa. Uberaba, MG, 2020.

\begin{tabular}{|c|c|c|c|c|}
\hline $\begin{array}{c}\text { AUTORES } \\
\text { PERIÓDICO (ANO) } \\
\text { TÍTULO }\end{array}$ & OBJETIVO & MÉTODOS & RESULTADOS / CONCLUSÃO & $\begin{array}{c}\text { NÍVEL DE } \\
\text { EVIDÊNCIA }\end{array}$ \\
\hline $\begin{array}{l}\text { Ratnakaran, } \\
\text { Prabhakaran } \quad \text { Karunakaran } \\
\text { Ka } \\
\text { Journal of Postgraduate } \\
\text { Medicine } \\
\text { (2016) } \\
\text { Prevalence of Burnout } \\
\text { and its correlates among } \\
\text { residents in a tertiary } \\
\text { medical center in Kerala, } \\
\text { India: A cross-sectional } \\
\text { study. }\end{array}$ & $\begin{array}{lr}\text { Estudar } & \mathrm{a} \\
\text { prevalência } & \text { de } \\
\text { Burnout e } & \text { suas } \\
\text { correlações } & \text { entre } \\
\text { estagiários } & \mathrm{e} \\
\text { residentes } & \text { na } \\
\text { Faculdade } & \text { de } \\
\text { Medicina } & \text { do } \\
\text { Governo } & \text { em } \\
\text { Thiruvanan- } \\
\text { thapuram, } \\
\text { Índia }\end{array}$ & $\begin{array}{l}\text { Trata-se de um estudo transversal de } 558 \\
\text { estagiários e residentes da Government } \\
\text { Medical College, Thiruvanan-thapuram, } \\
\text { Kerala, Índia. Foram coletados dados que } \\
\text { incluíram o Inventário de Burnout de } \\
\text { Copenhague [CBI] que avalia o Burnout nas } \\
\text { dimensões de Burnout Pessoal, Burnout de } \\
\text { Trabalho e Burnout Relacionado ao Paciente, } \\
\text { com uma pontuação de corte de } 50 \text { para cada } \\
\text { dimensão. }\end{array}$ & $\begin{array}{l}\text { O Burnout foi considerado o mais alto entre os estagiários nas } \\
\text { dimensões Burnout pessoal }(64,05 \%) \text { e Burnout relacionado ao } \\
\text { paciente }(68,62 \%) \text { e em residentes juniores para Burnout } \\
\text { relacionado ao trabalho }(40 \%) \text {. Os residentes sêniores super } \\
\text { especializados apresentaram a menor prevalência de Burnout nas } \\
\text { três dimensões. Entre os residentes, os residentes não } \\
\text { médicos/não cirúrgicos apresentaram menor prevalência de } \\
\text { Burnout nas três dimensões, enquanto os residentes com } \\
\text { especialidade cirúrgica apresentaram o maior Burnout pessoal } \\
(57,92 \%) \text { e Residentes com especialidade médica apresentaram o } \\
\text { maior Burnout relacionado ao paciente }(27,13 \%) \text {. Conclui-se que } \\
\text { a presença do Burnout foi observada em muitos residentes em } \\
\text { nosso estudo. }\end{array}$ & 4 \\
\hline $\begin{array}{l}\text { Fernandes, Beck, } \\
\text { Weiller, Viero, Freitas \& } \\
\text { Prestes }^{4} \\
\text { Revista Gaúcha de } \\
\text { Enfermagem } \\
\text { (2015) do prazer no } \\
\text { Sofrimento e pormação } \\
\text { processo de fesidentes } \\
\text { de em } \\
\text { multiprofissionais em } \\
\text { saúde. }\end{array}$ & $\begin{array}{lr}\text { Identificar } & \text { as } \\
\text { situações de prazer e } \\
\text { sofrimento } & \text { no } \\
\text { processo } & \text { de } \\
\text { formação } & \text { de } \\
\text { residentes } & \\
\text { multiprofissionais } \\
\text { em saúde. }\end{array}$ & $\begin{array}{l}\text { Pesquisa qualitativa, realizada em um } \\
\text { Programa de Residência Multiprofissional em } \\
\text { Saúde de uma universidade do sul do Brasil. } \\
\text { Os dados foram coletados em 2013, através } \\
\text { de grupos focais com nove residentes, e } \\
\text { analisados conforme a análise temática. }\end{array}$ & $\begin{array}{l}\text { As situações de sofrimento foram estímulos negativos dos } \\
\text { trabalhadores de saúde, dificuldades de participação em outras } \\
\text { atividades de formação profissional, excesso de atividades que os } \\
\text { residentes assumem como trabalhadores dos serviços de saúde, } \\
\text { falta de reconhecimento e dificuldades de integração das áreas da } \\
\text { Residência. As situações de prazer foram o desenvolvimento de } \\
\text { atividades multiprofissionais e possibilidade de aprendizado dos } \\
\text { residentes. Conclui-se que as situações de prazer e de sofrimento } \\
\text { identificadas podem auxiliar o planejamento de ações } \\
\text { institucionais que contribuam para um processo de formação } \\
\text { profissional que favoreça o aprendizado e o bem-estar dos } \\
\text { residentes. }\end{array}$ & 4 \\
\hline $\begin{array}{l}\text { Silva, Goulart, Lopes, } \\
\text { Serrano \& Guido } \\
\text { Revista de Enfermagem } \\
\text { da UFSM } \\
\text { (2014) } \\
\text { Estresse e hardiness } \\
\text { entre residentes } \\
\text { multiprofissionais de } \\
\text { uma universidade } \\
\text { pública. }\end{array}$ & $\begin{array}{l}\text { Verificar a } \\
\text { associação entre } \\
\text { estresse e Hardiness } \\
\text { em residentes } \\
\text { Multiprofissionais } \\
\text { de } \\
\text { Universidade } \\
\text { pública do Rio } \\
\text { Grande do Sul. }\end{array}$ & $\begin{array}{l}\text { Trata-se de um estudo analítico, transversal, } \\
\text { quantitativo. Aplicou-se um formulário de } \\
\text { dados sociodemográficos, a Escala de } \\
\text { Estresse no Trabalho e a Escala de Hardiness } \\
\text { em } 37 \text { residentes entre abril e junho de } 2011 \text {. } \\
\text { Valores de p<0,05 foram considerados } \\
\text { significativos. }\end{array}$ & $\begin{array}{l}\text { Observou-se que } 51,35 \% \text { dos residentes apresentam baixo } \\
\text { estresse e } 48,65 \% \text { alto estresse. A Personalidade Hardiness foi } \\
\text { verificada em } 24,32 \% \text { dos profissionais, e desses, } 21,62 \% \\
\text { apresentavam baixa intensidade de estresse. Houve correlação } \\
\text { significativa e negativa ( } \mathrm{p}=0,001, \mathrm{r}=-0,5105) \text { entre Hardiness e a } \\
\text { intensidade de estresse. Confirmou-se a hipótese de que } \\
\text { indivíduos Hardy apresentam menor intensidade de estresse. } \\
\text { Sugere-se a elaboração de estratégias de promoção de Hardiness } \\
\text { em vista de seus benefícios à saúde do residente. }\end{array}$ & 4 \\
\hline
\end{tabular}




\begin{tabular}{|c|c|c|c|c|}
\hline $\begin{array}{l}\text { Guido, Goulart, Silva, Lopes } \\
\text { \& Ferreira }{ }^{27} \\
\text { Revista Latino Americana } \\
\text { de Enfermagem } \\
\text { (2012) } \\
\text { Estresse e Burnout entre } \\
\text { residentes } \\
\text { multidisciplinares. }\end{array}$ & $\begin{array}{l}\text { Identificar associações } \\
\text { entre síndrome de alto } \\
\text { estresse e Burnout em } \\
\text { residentes } \\
\text { multidisciplinares de uma } \\
\text { universidade federal do Rio } \\
\text { Grande do Sul, Brasil. }\end{array}$ & $\begin{array}{l}\text { Trata-se de um estudo analítico, } \\
\text { transversal e quantitativo. } \\
\text { Questionários sociodemográficos e } \\
\text { a escala de estresse no trabalho e } \\
\text { pesquisa Maslach Burnout } \\
\text { Inventory - Health Services (MBI- } \\
\text { HSS) foram aplicados a } 37 \\
\text { residentes entre abril e junho de } \\
\text { 2011. P - valores <0,05 foram } \\
\text { considerados estatisticamente } \\
\text { significativos. }\end{array}$ & $\begin{array}{l}\text { Verificou-se que } 48,65 \% \text { dos residentes sofreram alto estresse. Ao } \\
\text { associar as subescalas do MBI-HSS, verificou-se que } 27 \% \text { dos } \\
\text { residentes apresentaram alguma indicação de síndrome de Burnout. } \\
\text { Houve correlação estatisticamente significativa }(\mathrm{p}=0,00, \mathrm{r}=0,68) \\
\text { entre alto estresse e Burnout. Conclui-se que o alto estresse foi } \\
\text { confirmado como sendo um preditor da síndrome de Burnout entre } \\
\text { residentes multidisciplinares. Por isso, propomos que sejam } \\
\text { realizados estudos de intervenção para mudar tais contextos. }\end{array}$ & 4 \\
\hline $\begin{array}{l}\text { Fink, Krugman, Casey \& } \\
\text { Goode }{ }^{28} \\
\text { Journal of Nursing } \\
\text { Administration - JONA } \\
\text { (2008) } \\
\text { The Graduate Nurse } \\
\text { Experience } \quad \text { Qualitative } \\
\text { Residency r Program } \\
\text { Outcomes. }\end{array}$ & $\begin{array}{l}\text { Analisar os discursos dos } \\
\text { entrevistados para } \\
\text { determinar se seus } \\
\text { comentários } \\
\text { enriqueceriam ainda mais } \\
\text { os dados quantitativos. } \\
\text { Determinar se a análise dos } \\
\text { temas extraídos dos dados } \\
\text { qualitativos poderia ser } \\
\text { usada para converter as } \\
\text { perguntas abertas do } \\
\text { instrumento em perguntas } \\
\text { quantitativas facilitando a } \\
\text { administração de teste e } \\
\text { procedimentos analíticos. }\end{array}$ & $\begin{array}{l}\text { Trata-se de um estudo qualitativo } \\
\text { realizado com enfermeiras } \\
\text { residentes em } 12 \text { locais de } \\
\text { hospitais acadêmicos no programa } \\
\text { de residência pós-bacharelado da } \\
\text { University Health System } \\
\text { Consortium (UHC)/American } \\
\text { Association of Colleges of Nursing } \\
\text { (AACN). Os participantes } \\
\text { responderam ao instrumento The } \\
\text { Casey-Fink Graduate Nurse } \\
\text { Experience Survey em três } \\
\text { períodos durante o primeiro ano de } \\
\text { Residência: no início, } 6 \text { meses e } 12 \\
\text { meses na conclusão do programa } \\
\text { de } 1 \text { ano. }\end{array}$ & $\begin{array}{l}\text { As análises qualitativas refletiram os desafios que os residentes de } \\
\text { enfermagem experimentaram durante a transição para a prática, } \\
\text { como: medo, falta de confiança e preocupações em prejudicar os } \\
\text { pacientes durante o } 1^{\circ} \text { ano de prática. A visibilidade, o apoio do } \\
\text { gerente de enfermagem, educadores ou residentes facilitadores para } \\
\text { treinar consistentemente o domínio das habilidades durante os } \\
\text { segundos } 6 \text { meses de prática, o apoio para integração na cultura da } \\
\text { unidade e da equipe são os principais achados desta pesquisa } \\
\text { qualitativa. Conclui-se que a análise qualitativa forneceu evidências } \\
\text { suficientes para converter perguntas abertas específicas no } \\
\text { instrumento Casey-Fink Graduate Nurse Experience Survey em um } \\
\text { formato quantitativo para facilidade de administração e análise. }\end{array}$ & 4 \\
\hline
\end{tabular}


No Quadro 3 são destacados os dados referentes aos fatores desencadeantes do estresse psicológico em residentes não médicos, de acordo com os respectivos estudos.

Quadro 3: Fatores desencadeantes do estresse psicológico em residentes não médicos. Uberaba, MG, 2020.

\begin{tabular}{|c|c|}
\hline FATORES DESENCADEANTES & ESTUDOS \\
\hline Sobrecarga de trabalho & $\begin{array}{l}\text { Fernandes, Beck, Weiller, Viero, Freitas \& Prestes }{ }^{4} \text {; Silva, Goulart, Lopes, } \\
\text { Serrano \& Guido } 8 \text {; Ratnakaran, Prabhakaran \& Karunakaran }{ }^{26} \text {; Guido, } \\
\text { Goulart, Silva, Lopes \& Ferreira }{ }^{27} \text {; Fink, Krugman, Casey \& Goode }{ }^{28} \text { (5) }\end{array}$ \\
\hline Atendimento a pacientes graves & $\begin{array}{l}\text { Silva, Goulart, Lopes, Serrano \& Guido }{ }^{8} \text {; Ratnakaran, Prabhakaran \& } \\
\text { Karunakaran }{ }^{26} \text {; Guido, Goulart, Silva, Lopes \& Ferreira }{ }^{27} \text {; Fink, Krugman, } \\
\text { Casey \& Goode }{ }^{28}(4)\end{array}$ \\
\hline Exaustão emocional & $\begin{array}{l}\text { Silva, Goulart, Lopes, Serrano \& Guido }{ }^{8} \text {; Guido, Goulart, Silva, Lopes \& } \\
\text { Ferreira }^{27} \text {; Fink, Krugman, Casey \& Goode }{ }^{28}(3)\end{array}$ \\
\hline Privação de sono & $\begin{array}{l}\text { Fernandes, Beck, Weiller, Viero, Freitas \& Prestes }{ }^{4} \text {; Silva, Goulart, Lopes, } \\
\text { Serrano \& Guido }{ }^{8} \text {; Guido, Goulart, Silva, Lopes \& Ferreira }{ }^{27} \text {; (3) }\end{array}$ \\
\hline Falta de reconhecimento & $\begin{array}{l}\text { Fernandes, Beck, Weiller, Viero, Freitas \& Prestes }{ }^{4} \text {; Fink, Krugman, } \\
\text { Casey \& Goode }{ }^{28}(2)\end{array}$ \\
\hline $\begin{array}{l}\text { Falta de orientação ou incerteza na } \\
\text { carreira }\end{array}$ & $\begin{array}{l}\text { Ratnakaran, Prabhakaran \& Karunakaran26; Fink, Krugman, Casey \& } \\
\text { Goode }^{28}(2)\end{array}$ \\
\hline Tipo do trabalho & $\begin{array}{l}\text { Ratnakaran, Prabhakaran \& Karunakaran }{ }^{26} \text {; Fink, Krugman, Casey \& } \\
\text { Goode }^{28}(2)\end{array}$ \\
\hline Alta Rotatividade entre os Setores & Ratnakaran, Prabhakaran \& Karunakaran ${ }^{26}(1)$ \\
\hline Emergências no trabalho & Ratnakaran, Prabhakaran \& Karunakaran ${ }^{26}(1)$ \\
\hline Engajamento no trabalho & Ratnakaran, Prabhakaran \& Karunakaran ${ }^{26}(1)$ \\
\hline $\begin{array}{l}\text { Falta de participação nas tomadas de } \\
\text { decisões }\end{array}$ & Ratnakaran, Prabhakaran \& Karunakaran ${ }^{26}$ (1) \\
\hline Nível na carreira & Ratnakaran, Prabhakaran \& Karunakaran ${ }^{26}(1)$ \\
\hline Tempo de trabalho & Ratnakaran, Prabhakaran \& Karunakaran ${ }^{26}(1)$ \\
\hline Falta de experiência e habilidades & Fink, Krugman, Casey \& Goode ${ }^{28}(1)$ \\
\hline
\end{tabular}

\section{DISCUSSÃO}

Os estudos considerados foram publicados entre 2008 e 2016, em português e inglês, em cinco periódicos diferentes e apresentaram nível de evidência 4. Esses dados devem implicar no incentivo para se desenvolver estudos de melhor delineamento metodológico, principalmente experimentais, no intuito de explorar o estresse psicológico em residentes não médicos, possibilitando uma decisão clínica baseada em evidências mais confiáveis ${ }^{29}$.

Nesta perspectiva, identificou-se os fatores que desencadeiam o desenvolvimento do estresse psicológico em residentes não médicos. Dentre os fatores mais citados que desencadeiam o estresse destacaram-se: a sobrecarga de trabalho, o atendimento a pacientes graves, exaustão emocional e privação de sono ${ }^{4,8,26-28}$.

Pesquisas realizadas em universidades do sul do Brasil com residentes multiprofissionais apontaram a sobrecarga relacionada à excessiva carga de trabalho assistencial como causa do estresse. Essa relação deve-se a transição das responsabilidades e pressões profissionais que o residente assume $8,27$. 
Outro estudo identificado com residentes vinculados a um Programa de Residência Multiprofissional no sul do Brasil aborda o acumulo de funções assistenciais como desencadeador da Síndrome de Burnout, justificada pelo fato de extrapolar-se o expediente para cumprir todas as atividades que lhes foram designadas e apesar de elevar seus esforços acabam muitas vezes frustrados por não conseguirem ${ }^{4}$.

Uma outra pesquisa realizada em uma universidade do Paraná, Brasil, com residentes reforça a alta demanda de atividades que interfere negativamente em suas vidas, causando-lhes o estresse ${ }^{7}$. 0 acúmulo das funções muitas vezes dar-se-á pela falta de tempo para planejar as atividades, inexperiência em rotinas de trabalho, bem como pela falta de clareza nas atribuições aos residentes ${ }^{7}$.

Um estudo realizado com internos e residentes do Government Medical College no sul da Índia aponta o atendimento a pacientes graves como um fator desencadeador do estresse e se justifica pela necessidade de prestar um cuidado intensivo, devido à constante instabilidade do paciente grave e até mesmo pelo fato de que muitas vezes os residentes podem se sentir despreparados frente à assistência prestada a esses pacientes ${ }^{26}$.

Outra investigação realizada em um hospital universitário do Paraná, Brasil, com residentes multiprofissionais, destacou que os residentes vivem em regime de estresse nas unidades com pacientes extremamente críticos, assim como naqueles setores onde os pacientes são admitidos entre a vida e a morte ${ }^{30}$.

Uma pesquisa realizada com profissionais de Unidades de Terapia Intensiva (UTI) em um hospital de multiespecialidades no sul da Índia, destacou a gravidade no quadro do paciente como fator do estresse psicológico dos profissionais da saúde, pois a assistência prestada nas UTIs requer maior atenção dos profissionais e, em caso de piora clínica do paciente, há uma atuação imediata que desencadeia o estresse, além dos profissionais estarem constantemente em contato com o sofrimento e morte 31 .

Nos achados desta revisão a privação do sono foi outro fator que desencadeia o estresse $^{4,8,27}$ que corrobora um trabalho em Singapura, que traz a extensa carga horaria dedicada ao trabalho e aos estudos que por sua vez acabam por restringir os momentos de lazer dos residentes ${ }^{32}$.

Um estudo realizado em um hospital de ensino em Belo Horizonte, com residentes de cirurgia vascular evidenciou o estresse psicológico relatado por residentes que expõem noites mal dormidas ou poucas horas de sono, assim como medo de retaliação e assédio moral sofrido durante o período da residência33.

No Brasil, outros estudos realizados com residentes médicos e não médicos e uma revisão narrativa sobre evolução conceitual do estresse evidenciaram que a privação e má qualidade do sono são fatores que interferem na saúde psicológica das pessoas, causando estresse, depressão, ansiedade e sinais e sintomas da Síndrome de Burnout ${ }^{9,34,35}$.

Uma pesquisa realizada com residentes em uma maternidade do Rio Grande do Norte retratou que eles possuem sintomas psicológicos, tais como cansaço excessivo, vontade de fugir de tudo, angústia, ansiedade diária e dúvida quanto a si próprios, o que sinaliza sofrimento psíquico e problemas na saúde decorrentes da sobrecarga de trabalho, atendimento a pacientes graves e privação de sono ${ }^{14}$.

0 estresse psicológico em residentes não médicos possui inúmeros fatores desencadeantes que vão desde o atendimento a pacientes graves, o acúmulo de funções e atividades a sobrecarga de trabalho, que pode ser traduzida pela carga horária excessiva, complexidade do trabalho e pressão, falta de recursos humanos, não colaboração dos colegas, distribuição inadequada de tarefas, exaustão emocional até a privação de sono ${ }^{36}$.

Estudos que identificam os fatores estressores que afetam os residentes não médicos são importantes, para que, se baseando nessas causas os programas de Residência, juntamente com as Universidades, proponham estratégias e ações que visem a redução do estresse nesta população, melhorando a qualidade de vida destes profissionais de saúde ${ }^{14}$. 


\section{CONCLUSÃO}

Das pesquisas selecionadas emergiram os fatores desencadeantes do estresse psicológico como: sobrecarga de trabalho, o atendimento a pacientes graves, exaustão emocional e privação de sono.

O número inicial de artigos foi grande, porém a maioria focava em residentes médicos e estudantes de medicina, tanto nas produções nacionais como a internacionais, mostrando poucas evidências cientificas sobre os fatores desencadeantes do estresse psicológico nos residentes não médicos, sendo esta uma importante limitação da pesquisa. Portanto é indispensável a elaboração de estudos científicos bem delineados, com bom nível de evidência, que explorem a temática com maior profundidade e verifiquem os fatores desencadeantes do estresse psicológico.

\section{REFERÊNCIAS}

1. Silva LS, Natal S. Residência multiprofissional em saúde: análise da implantação de dois programas pela Universidade Federal de Santa Catarina, Brasil. Trab Educ Saúde [Internet]. 2019 [citado em 26 maio 2020]; 17(3):e0022050. Disponível em: https://www.scielo.br/j/tes/a/s5N35mz7j9wphWnHp8bW6wJ/?lang=pt. DOI: https://doi.org/10.1590/1981-7746-sol00220

2. Silva LB. Residência multiprofissional em saúde no Brasil: alguns aspectos da trajetória histórica. Rev Katálysis [Internet]. 2018 [citado em 26 maio 2020]; 21(1):200-9. Disponível em: https://www.scielo.br/j/rk/a/BpFH8tww34qhgm9LSW6n84d/?lang=pt. DOI: https://doi.org/10.1590/1982-02592018v21n1p200

3. Rocha JS, Casarotto RA, Schmitt ACB. Saúde e trabalho de residentes multiprofissionais. Rev Cienc Salud (Bogotá) [Internet]. 2018 [citado em 15 fev 2020]; 16(3):447-62. Disponível em: http://www.scielo.org.co/scielo.php?script=sci_abstract\&pid=S1692-

$72732018000300447 \& \operatorname{lng}=$ en\&nrm=iso\&tlng=pt.

DOI: https://doi.org/10.12804/revistas.urosario.edu.co/revsalud/a.7265

4. Fernandes MNS, Beck CLC, Weiller TH, Viero V, Freitas PH, Prestes FC. Suffering and pleasure in the process of forming multidisciplinary health residents. Rev Gaúch Enferm. [Internet]. 2015 [citado em 17 fev 2020]; 36(4):90-7. Disponível em: https://www.scielo.br/j/rgenf/a/WXFK3LZ7yPp3QChRsdkGtDN/?lang=en. DOI: https://doi.org/10.1590/1983-1447.2015.04.50300

5. Aldubai SAR, Aljohani AM, Alghamdi AG, Alghamdi KS, Ganasegeran K, Yenbaawi AM. Prevalence and associated factors of burnout among family medicine residents in Al Madina, Saudi Arabia. J Family Med Prim Care [Internet]. 2019 [citado em 26 maio 2020]; 8(2):657-62. Disponível em: https://www.jfmpc.com/article.asp?issn=2249-

4863; year $=2019$; volume $=8$;issue $=2$; spage $=657$; epage $=662$; aulast $=$ Aldubai .

DOI: https://doi.org/10.4103/jfmpc.jfmpc_268_18

6. Zubairi AJ, Noordin S. Factors associated with burnout among residents in a developing country. Ann Med Surg (Lond) [Internet]. 2016 [citado em 26 maio 2020]; 6:60-3. Disponível em: https://www.sciencedirect.com/science/article/pii/S2049080116000947?via\%3Dihub. $\quad$ DOI: https://doi.org/10.1016/j.amsu.2016.01.090

7. Balan KCK, Jorge IMP, Silva DB. Avaliação do nível de estresse ocupacional em residentes de um programa de residência multiprofissional em saúde. Rev Bras Inov Tecnol Saúde [Internet]. 2018 [citado em 16 abr 2020]; 8(1):51-66. Disponível em: https://periodicos.ufrn.br/reb/article/view/13631. DOI: https://doi.org/10.18816/r-bits.v8i1.13631

8. Silva RM, Goulart CT, Lopes LFD, Serrano PM, Guido LA. Estresse e hardiness entre residentes multiprofissionais de uma universidade pública. Rev Enferm UFSM [Internet]. 2014 [citado em 13 fev 2020]; 4(1):87-96. Disponível em: https://periodicos.ufsm.br/index.php/reufsm/article/view/8921. DOI: https://doi.org/10.5902/217976928921

9. Silva RM, Goulart CT, Guido LA. Evolução histórica do conceito de estresse. REVISA (Online) [Internet]. 2018 [citado em 13 fev 2020]; 7(2):148-56. Disponível em: http://revistafacesa.senaaires.com.br/index.php/revisa/article/view/316/225 
10. Kam SXL, Toledo ALS, Pacheco CC, Souza GFB, Santana VLM, Bonfá-Araújo B, et al. Estresse em estudantes ao longo da graduação médica. Rev Bras Educ Méd. [Internet]. 2019 [citado em 02 jun 2020]; 43(Supl1):246-53. Disponível em: https://www.scielo.br/j/rbem/a/3Q4H6dQLkXCVwgQqMSZqfmk/?lang=pt.

DOI: https://doi.org/10.1590/1981-5271v43suplemento1-20180192

11. Silveira L, Bez CN, Gonçalves Júnior E, Peiter CC, Fabrizzio GC, Santos JLG. Síndrome de Burnout em preceptores e residentes vinculados a programas de residência em saúde da família. Cogitare Enferm. [Internet]. 2020 [citado em 02 jun 2020]; 25:e6724. Disponível em: https://revistas.ufpr.br/cogitare/article/view/67248. DOI: http://dx.doi.org/10.5380/ce.v25i0.67248 12. Bezerra CM, Assis SG, Constantino P. Sofrimento psíquico e estresse no trabalho de agentes penitenciários: uma revisão da literatura. Ciênc Saúde Colet. [Internet]. 2016 [citado em 02 jun 2020]; 21(7):2135-46. Disponível em: https://www.scielo.br/j/csc/a/QPHfzKLf87DFPkxRgxZsNRd/?lang=pt. DOI: https://doi.org/10.1590/1413-81232015217.00502016

13. Bordin D, Feltrin F, Cabral LPA, Fadel CB. Impacto do estresse na qualidade de vida e condutas de saúde de residentes multiprofissionais. Rev Gest Sist Saúde [Internet]. 2019 [citado em 13 fev 2020]; 8(3):385-94. Disponível em: https://periodicos.uninove.br/revistargss/article/view/14928. DOI: https://doi.org/10.5585/rgss.v8i3.14928

14. Silva RMB, Moreira SNT. Estresse e residência multiprofissional em saúde: compreendendo significados no processo de formação. Rev Bras Educ Méd. [Internet]. 2019 [citado em 03 fev 2020]; 43(4):157-66. Disponível

https://www.scielo.br/j/rbem/a/xdRBHKycxFSV3jtFMDZYhxS/?lang=pt.

em: http://dx.doi.org/10.1590/1981-52712015v43n4rb20190031

15. Pomini MC, Bordin D, Saliba NA, Momaz SAS, Fadel CB. A influência da posição acadêmica sobre condutas de saúde em universitários. Rev ABENO [Internet]. 2018 [citado em 05 fev 2020]; 18(1):7483. Disponível em: https://revabeno.emnuvens.com.br/revabeno/article/view/395/372. DOI: https://doi.org/10.30979/rev.abeno.v18i1.395

16. Valério RL, Oliveira EB, Kestenberg CCF, Paula VG, Dias LBS, Oliveira TS. Emotional exhaustion in resident nurses of specialized units in a university hospital. Res Soc Develop. [Internet]. 2020 [citado em 01 jun 2020]; 9(2):1-18. Disponível em: https://rsdjournal.org/index.php/rsd/article/view/2240. DOI: https://doi.org/10.33448/rsd-v9i2.2240

17. Silva DS, Merces MC, Souza MC, Gomes AMT, Lago SB, Beltrame M. Síndrome de Burnout em residentes multiprofissionais em saúde. Rev Enferm UERJ [Internet]. 2019 [citado em 26 maio 2020]; 27:e43737. Disponível em: https://www.epublicacoes.uerj.br/index.php/enfermagemuerj/article/view/43737. DOI: https://doi.org/10.12957/reuerj.2019.43737

18. Goulart CT, Guido LA, Silva RM, Bublitz S, Grazziano ES. Efeitos dos programas de manejo de estresse na saúde do trabalhador: revisão integrativa. Rev Enferm UFPE on line [Internet]. 2015 [citado em 14 maio 2020]; 9(4):7431-8. DOI: 10.5205/reuol.7275-62744-1-SM.0904201531

19. Graça CC, Zagonel IPS. Estratégias de coping e estresse ocupacional em profissionais de enfermagem: revisão integrativa. Espaço Saúde (Online) [Internet]. 2019 [citado em 28 maio 2020]; 20(2):67-77.

Disponível

http://espacoparasaude.fpp.edu.br/index.php/espacosaude/article/view/622/pdf.

em: https://doi.org/10.22421/15177130-2019v20n2p67

20. Mendes KDS, Silveira RCCP, Galvão CM. Revisão integrativa: método de pesquisa para a incorporação de evidências na saúde e na enfermagem. Texto \& Contexto Enferm. [Internet]. 2008 [citado em 02 fev 2020]; 17(4):758-64. Disponível em: https://www.scielo.br/j/tce/a/XzFkq6tjWs4wHNqNjKJLkXQ/?lang=pt. DOI: http://dx.doi.org/10.1590/S0104-07072008000400018

21. Moher D, Liberati A, Tetzlaff J, Altman DG, The PRISMA Group. Preferred reporting items for systematic reviews and metanalyses: the PRISMA statement. PLoS Med. [Internet]. 2009 [citado em 07 fev 2020]; 6(7):e1000097. Disponível em: https://journals.plos.org/plosmedicine/article?id=10.1371/journal.pmed.1000097. DOI: https://doi.org/10.1371/journal.pmed.1000097

22. Sousa LMM, Marques JM, Firmino CF, Frade F, Valentim OS, Antunes AV. Modelos de formulação da questão de investigação na prática baseada na evidência. Rev Invest Enferm. [Internet]. 2018 [citado 30 jun 2020]; (23):31-9. Disponível em: http://hdl.handle.net/20.500.12253/1287. 
23. Ouzzani M, Hammady H, Fedorowicz Z, Elmagarmid A. Rayyan - a web and mobile app for systematic reviews. Syst Rev. [Internet]. 2016 [citado em 16 fev 2020]; 5(1):210. Disponível em: https://systematicreviewsjournal.biomedcentral.com/articles/10.1186/s13643-016-0384-4. DOI: https://doi.org/10.1186/s13643-016-0384-4

24. Ursi ES, Galvão CM. Prevenção de lesões de pele no perioperatório: revisão integrativa da literatura. Rev Latinoam Enferm. [Internet]. 2006 [citado em 02 fev 2020]; 14(1):124-31. Disponível em: https://www.scielo.br/j/rlae/a/7hS3VgZvTs49LNX9dd85VVb/?lang=pt.

DOI: https://doi.org/10.1590/S0104-11692006000100017

25. Agency for Healthcare Research and Quality. Quality Improvement and monitoring at your fingertips [Internet]. Rockville, MD: AHRQ; 2016 [citado em 30 jun 2020]. Disponível em: http://www.qualityindicators.ahrq.gov

26. Ratnakaran B, Prabhakaran A, Karunakaran V. Prevalence of burnout and its correlates among residents in a tertiary medical center in Kerala, India. J Postgrad Med. [Internet]. 2016 [citado em 17 fev 2020]; 62(3):157-61. Disponível em: https://www.ncbi.nlm.nih.gov/pmc/articles/PMC4970341/. DOI: https://doi.org/ 10.4103/0022-3859.184274

27. Guido LA, Goulart CT, Silva RM, Lopes LFD, Ferreira, EM. Stress and Burnout among multidisciplinary residents. Rev Latinoam Enferm. [Internet]. 2012 [citado em 17 fev 2020]; 20(6):1064-71.

Disponível

em:

https://www.scielo.br/j/rlae/a/LCq7bzR76RjDsKvpVYDhkCp/?lang=en.

https://doi.org/10.1590/S0104-11692012000600008

28. Fink R, Krugman M, Casey K, Goode C. The Graduate nurse experience qualitative residency program outcomes. J Nurs Adm. [Internet]. 2008 [citado em 17 fev 2020]; 38(7/8):341-8. Disponível em: https://journals.lww.com/jonajournal/Abstract/2008/07000/The_Graduate_Nurse_Experience_Qual itative.7.aspx. DOI: https://doi.org/10.1097/01.NNA.0000323943.82016.48

29. Dutra HS, Reis VN. Desenhos de estudos experimentais e quase-experimentais: definições e desafios na pesquisa em enfermagem. Rev Enferm UFPE on line [Internet]. 2016 [citado em 14 maio 2020]; 10(6):2230-41. Disponível em: https://periodicos.ufpe.br/revistas/revistaenfermagem/article/view/11238. DOI: https://doi.org/10.5205/ reuol.9199-80250-1-SM1006201639

30. Oliveira EB, Zanesco C, Bordin D, Cabral LPA, Brasil D, Fadel CB. Estresse na residência multiprofissional em saúde: natureza e magnitude. Braz J Dev. [Internet]. 2019 [citado em 14 maio 2020]; 5(11):25684-97. Disponível em: https://www.brazilianjournals.com/index.php/BRJD/article/view/4724/4355. DOI: https://doi.org/10.34117/bjdv5n11-225

31. Saravanabavan L, Sivakumar MN, Hisham M. Stress and Burnout among intensive care unit healthcare professionals in an indian tertiary care hospital. Indian J Crit Care Med. [Internet]. 2019 [citado em 14 maio 2020]; 23(10):462-6. Disponível em: https://www.ijccm.org/doi/pdf/10.5005/jpjournals-10071-23265. DOI: https://doi.org/10.5005/jp-journals-10071-23265

32. Chaou CH, Chang YC, Yu SR, Tseng HM, Hsiao CT, Wu KH, et al. Sleep, activity and fatigue reported by Postgraduate Year 1 residents: a prospective cohort study comparing the effects of night float versus the traditional overnight on-call system. Singapore Med J. [Internet]. 2018 [citado em 10 nov 2020]; 59(12):652-5. Disponível em: http://www.smj.org.sg/article/sleep-activity-and-fatigue-reportedpostgraduate-year-1-residents-prospective-cohort-study.

https://doi.org/10.11622/smedj.2018036

DOI:

33. Azevedo ARI, Rocha MR, Rezende AML, Vieira JV, Sousa RC, Gonçalves ALCP. Um estudo de caso sobre estresse em residentes de cirurgia vascular periférica em um hospital de ensino. Braz J Dev. [Internet]. 2020 [citado em 06 nov 2020]; 6(4):19475-96. Disponível em: https://www.brazilianjournals.com/index.php/BRJD/article/view/8781/7522. DOI: https://doi.org/10.34117/bjdv6n4-205

34. Kobayasi R. Qualidade de vida, resiliência, empatia, sonolência diurna e desempenho acadêmico em residentes de clínica médica: análise qualitativa e quantitativa [Internet]. [dissertação]. São Paulo: Universidade de São Paulo; 2018 [citado em 19 mar 2020]. 152p. Disponível em: https://teses.usp.br/teses/disponiveis/5/5169/tde-02082018-114511/pt-br.php. DOI: https://doi.org/10.11606/T.5.2018.tde-02082018-114511

35. Negreiros CTF, Silva SRS, Silva RM, Costa ALS, Farias FC, Cangussu DDD et al. Associação da qualidade do sono e perfil acadêmico com o estresse de estudantes de enfermagem. Rev Baiana Enferm. 
[Internet]. 2019 [citado em 20 abr 2020]; 33:e33482. Disponível em: https://periodicos.ufba.br/index.php/enfermagem/article/view/33482. DOI: https://doi.org/10.18471/rbe.v33.33482

36. Santos NAR, Santos J, Silva VR, Passos JP. Estresse ocupacional na assistência de cuidados paliativos em oncologia. Cogitare Enferm. [Internet]. 2017 [citado em 20 abr 2020]; 22(4):e50686. Disponível em: https://revistas.ufpr.br/cogitare/article/view/50686. DOI: http://dx.doi.org/10.5380/ce.v22i4.50686

Editora Associada: Fernanda Carolina Camargo

\section{CONTRIBUIÇÕES}

Gabriela França Rosinha e Fabiana Cristina Pires atuaram na concepção do estudo e seu projeto, coleta e análise dos dados, redação. Suzel Regina Ribeiro Chavaglia contribuiu na concepção, redação e revisão. Rosali Isabel Barduchi Ohl, Lúcia Aparecida Ferreira e Álvaro da Silva Santos participaram na revisão.

\section{Como citar este artigo (Vancouver)}

Rosinha GF, Chavaglia SRR, Pires FC, Ohl RIB, Ferreira LA, Santos ÁS. Fatores desencadeantes do estresse psicológico em residentes não médicos: revisão integrativa. REFACS [Internet]. 2021 [citado em inserir dia, mês e ano de acesso]; 9(4):1002-14. Disponível em: inserir link de acesso. DOI: inserir link do DOI.

\section{Como citar este artigo (ABNT)}

ROSINHA, G. F.; CHAVAGLIA, S. R. R.; PIRES, F. C.; OHL, R. I. B.; FERREIRA, L. A.; SANTOS Á. S. Fatores desencadeantes do estresse psicológico em residentes não médicos: revisão integrativa. REFACS, Uberaba, MG, v. 9, n. 4, p. 1002-14, 2021. Disponível em: inserir link de acesso. Acesso em: inserir dia, mês e ano de acesso. DOI: inserir link do DOI.

\section{Como citar este artigo (APA)}

Rosinha, G.F., Chavaglia, S.R.R., Pires, F.C., Ohl, R.I.B., Ferreira, L.A., \& Santos, Á.S. (2021). Fatores desencadeantes do estresse psicológico em residentes não médicos: revisão integrativa. REFACS, 9(4), 1002-14. Recuperado em: inserir dia, mês e ano de acesso de inserir link de acesso. DOI: inserir link do DOI. 